\title{
A Class of Laguerre-Based Generalized Humbert Polynomials
}

\author{
Saniya Batra $(\mathbb{D})$ and Prakriti Rai \\ Department of Mathematics, Amity University, Noida 201313, Utter Pradesh, India \\ Correspondence should be addressed to Saniya Batra; saniyabatra8@gmail.com
}

Received 19 May 2021; Revised 9 July 2021; Accepted 13 July 2021; Published 30 July 2021

Academic Editor: Patricia J. Y. Wong

Copyright (c) 2021 Saniya Batra and Prakriti Rai. This is an open access article distributed under the Creative Commons Attribution License, which permits unrestricted use, distribution, and reproduction in any medium, provided the original work is properly cited.

Several mathematicians have extensively investigated polynomials, their extensions, and their applications in various other research areas for a decade. Our paper aims to introduce another such polynomial, namely, Laguerre-based generalized Humbert polynomial, and investigate its properties. In particular, it derives elementary identities, recursive differential relations, additional symmetry identities, and implicit summation formulas.

\section{Introduction}

In all the given definitions, let $\mathbb{C}, \mathbb{R}, \mathbb{R}^{+}$, and $\mathbb{N}$ be the sets of complex numbers, real numbers, positive real numbers, and natural numbers, respectively.

The two-variable Kampé de Fériet generalized Hermite polynomial (see [1]) is defined as

$$
e^{x t+y t^{2}}=\sum_{n=0}^{\infty} H_{n}(x, y) \frac{t^{n}}{n !} .
$$

The finite series representation of Hermite polynomial of two variables is given by

$$
H_{n}(x, y)=n ! \sum_{r=0}^{[n / 2]} \frac{(y)^{r}(x)^{n-2 r}}{r !(n-2 r) !} .
$$

Substituting $y=-1$ and replacing $x$ by $2 x$, the polynomial in equation (2) reduces to ordinary Hermite polynomial (see $[1,2])$.

Classical Laguerre polynomial and its orthogonality $[3,4]$ have been studied extensively. Its generalization is given by the two variable Laguerre polynomial. The twovariable Laguerre polynomial $L_{n}(x, y)$ is defined by the following generating function (see [5-8]):

$$
e^{y t} C_{0}(x t)=\sum_{n=0}^{\infty} L_{n}(x, y) \frac{t^{n}}{n !},
$$

where $C_{0}(x)$ is the 0 -th order Tricomi function (see $[2,6-8])$ :

$$
C_{0}(x)=\sum_{r=0}^{\infty} \frac{(-1)^{r} x^{r}}{(r !)^{2}}
$$

The explicit expression of two-variable Laguerre polynomial is given as

$$
L_{n}(x, y)=\sum_{k=0}^{n} \frac{n !(-1)^{k} y^{n-k} x^{k}}{(n-k) !(k !)^{2}}, \quad\left(n \in \mathbb{N}_{0}\right) .
$$

We would now recall the following well-known generating functions, which will be further used in our paper:

$$
\left(1-2 x t+t^{2}\right)^{-1 / 2}=\sum_{n=0}^{\infty} P_{n}(x) t^{n},
$$

where $P_{n}(x)$ is the Legendre polynomial of first kind. Also,

$$
\left(1-2 x t+t^{2}\right)^{-1}=\sum_{n=0}^{\infty} U_{n}(x) t^{n}
$$

where $U_{n}(x)$ is the Chebyshev polynomial of the second kind.

The following generating function gives the extension of equations (6) and (7):

$$
\left(1-2 x t+t^{2}\right)^{-v}=\sum_{n=0}^{\infty} C_{n}^{v}(x) t^{n}
$$

where $C_{n}^{\nu}(x)$ is Gegenbauer polynomial. 
Substituting $v=1 / 2$ and $v=1$, equation (8) reduces to Legendre polynomial and Chebyshev polynomial, respectively:

$$
\left(1-m x t+t^{m}\right)^{-v}=\sum_{n=0}^{\infty} h_{n, m}^{v}(x) t^{n}
$$

where $h_{n, m}^{v}(x)$ is the Humbert polynomial defined as

$$
h_{n, m}^{v}(x)=\sum_{k=0}^{[n / m]} \frac{(-1)^{k}(\nu)_{n+(1-m) k}(m x)^{n-m k}}{k !(n-m k) !},
$$

where $m$ is a positive integer.

In 1991, another generalization was given by Milovanović and Djordjević (see [9]), which has the following generating function:

$$
\left(1-2 x t+t^{m}\right)^{-\lambda}=\sum_{n=0}^{\infty} p_{n, m}^{\lambda}(x) t^{n}
$$

where $m \in \mathbb{N}$ and $\lambda>-1 / 2$. Also,

$$
p_{n, m}^{\lambda}(x)=\sum_{k=0}^{[n / m]} \frac{(-1)^{k}(\lambda)_{n-(m-1) k}(2 x)^{n-m k}}{k !(n-m k) !} .
$$

Generalization of two variables of all the above polynomials and many more was given by Djordjević (see [10]) in the form

$$
\left(1-2(x+y) t+t^{m}(2 x y+1)\right)^{-\alpha}=\sum_{n=0}^{\infty} G_{n}^{\alpha, m}(x, y) t^{n},
$$

where

$$
G_{n}^{\alpha, m}(x, y)=\sum_{k=0}^{[n / m]} \frac{(-1)^{k}(\alpha)_{n-(m-1) k}(2(x+y))^{n-m k}(2 x y+1)^{k}}{k !(n-m k) !} .
$$

For $\alpha=1$ and $=1 / 2$, the above polynomial reduces to Chebyshev polynomial of two variables, $U_{n}^{m}(x, y)$, and Legendre polynomial of two variables, $P_{n}^{m}(x, y)$, respectively.

For $m=2$ and $y=0$, the above polynomial reduces to Gegenbauer polynomial.

Furthermore, by substituting $y=0$, the above polynomial reduces to $p_{n, m}^{\lambda}(x)$, the polynomial defined by Milovanović and Djordjević (see [9]).
The three-variable Hermite-Laguerre polynomial ${ }_{H} L_{n}(x, y, z)$ is defined by the following generating function (see [6]):

$$
e^{y t+z t^{2}} C_{0}(x t)=\sum_{n=0}^{\infty}{ }_{H} L_{n}(x, y, z) \frac{t^{n}}{n !}
$$

In our paper, we will introduce Laguerre-based generalized Humbert polynomials ${ }_{L} G_{n}^{v, m}(a, b, c ; x, y, z)$.

Definition 1. The Laguerre-based generalized Humbert polynomials of order $v$, denoted by ${ }_{L} G_{n}^{v, m}(a, b, c ; x, y, z)$, is defined by the following generating function:

$$
\begin{aligned}
{[1} & \left.-2(a+b) t+t^{m}(2 a b+1)\right]^{-v} c^{y t+z t^{2}} C_{0}(x t) \\
& =\sum_{n=0}^{\infty}{ }_{L} G_{n}^{v, m}(a, b, c ; x, y, z) t^{n}
\end{aligned}
$$

where $a, b, c, x, y, z, v \in \mathbb{C}$ and $m \in \mathbb{N}$.

For all the further work, let

$$
{ }_{L} G_{n}^{v, m}(a, b, e ; x, y, z)={ }_{L} G_{n}^{v, m}(a, b ; x, y, z) .
$$

\section{Elementary Identities of ${ }_{\mathbf{L}} \mathbf{G}_{\mathbf{n}}^{v, \mathbf{m}}(\mathbf{a}, \mathbf{b}, \mathbf{c} ; \mathbf{x}, \mathbf{y}, \mathbf{z})$}

For our further reference, let us recall the following identities mentioned in the lemma as follows (see $[11,12]$ ).

Lemma 1. The following relations hold:

$$
\begin{aligned}
& \sum_{n=0}^{\infty} \sum_{m=0}^{\infty} A_{m, n}=\sum_{n=0}^{\infty} \sum_{m=0}^{[n / k]} A_{m, n-k m}, \\
& \sum_{n=0}^{\infty} \sum_{m=0}^{[n / k]} A_{m, n}=\sum_{n=0}^{\infty} \sum_{m=0}^{\infty} A_{m, n+k m},
\end{aligned}
$$

$$
\sum_{N=0}^{\infty} f(N) \frac{(x+y)^{N}}{N !}=\sum_{m, n=0}^{\infty} f(m+n) \frac{x^{m}}{m !} \frac{y^{n}}{n !},
$$

where $f(N)$ and $A_{m, n}$ are complex-and real-valued functions with $m, n, N \in \mathbb{N}_{0}$ and $x, y \in \mathbb{C}$. Lemma 1 applies to the convergent double series.

Theorem 1. For $a, b, c, x, y, z, v \in \mathbb{C}$ and $m \in \mathbb{N}$, the following relations are satisfied:

$$
\begin{aligned}
& \frac{\partial^{k}}{\partial y^{k} L} G_{n}^{v, m}(a, b, c ; x, y, z)=\log ^{k} c_{L} G_{n-k}^{v, m}(a, b, c ; x, y, z), \quad\left(n, k \in \mathbb{N}_{0} ; k \leq n\right), \\
& \frac{\partial^{k}}{\partial z^{k} L} G_{n}^{v, m}(a, b, c ; x, y, z)=\log ^{k} c_{L} G_{n-2 k}^{v, m}(a, b, c ; x, y, z), \quad\left(n, k \in \mathbb{N}_{0} ; 2 k \leq n\right),
\end{aligned}
$$




$$
\begin{aligned}
& \frac{\partial^{k+l}}{\partial y^{k} \partial z^{l}} G_{n}^{v, m}(a, b, c ; x, y, z)=\frac{\partial^{k+l}}{\partial z^{l} \partial y^{k}} G_{n}^{v, m}(a, b, c ; x, y, z)=\log ^{k+l} c_{L} G_{n-k-2 l}^{v, m}(a, b, c ; x, y, z), \quad\left(n, k \in \mathbb{N}_{0} ; k+2 l \leq n\right), \\
& { }_{L} G_{n}^{v, m}(a, b ; x, y, z)=\sum_{r=0}^{n} \frac{G_{n-r}^{v, m}(a, b){ }_{H} L_{r}(x, y, z)}{r !}, \quad\left(n \in \mathbb{N}_{0}\right), \\
& { }_{L} G_{n}^{v, m}(a, b ; x, y, z)=\frac{1}{n !} \sum_{k=0}^{n} \sum_{l=0}^{k}\left(\begin{array}{l}
n \\
k
\end{array}\right)\left(\begin{array}{l}
k \\
l
\end{array}\right) \frac{l !(-x)^{n-k}}{(n-k) !} H_{k-l}(x, y) G_{l}^{v, m}(a, b), \quad\left(n \in \mathbb{N}_{0}\right), \\
& { }_{L} G_{n}^{v, m}(a, b ; x, y, z)=\sum_{k=0}^{n} \sum_{l=0}^{[k / 2]} \frac{z^{l}}{(k-2 l) ! l !} G_{n-k}^{v, m}(a, b) L_{k-2 l}(x, y), \quad\left(n \in \mathbb{N}_{0}\right), \\
& { }_{H} L_{n}(x, y, z)=\sum_{k=0}^{n} G_{n-k}^{-v, m}(a, b)_{L} G_{n}^{v, m}(a, b ; x, y, z), \quad\left(n \in \mathbb{N}_{0}\right), \\
& (-1)^{n} x^{n}=\sum_{k=0}^{n} \sum_{l=0}^{k}\left(\begin{array}{l}
n \\
k
\end{array}\right) k ! H_{n-k}(-y,-z) G_{k-l}^{-v, m}(a, b)_{L} G_{l}^{v, m}(a, b ; x, y, z), \\
& =n ! \sum_{k=0}^{n} \sum_{l=0}^{k} \frac{G_{n-k}^{-\nu, m}(a, b) H_{k-l}(-y,-z){ }_{L} G_{l}^{\nu, m}(a, b ; x, y, z)}{(k-l) !}, \\
& =\sum_{k=0}^{n} \sum_{l=0}^{k}\left(\begin{array}{c}
n \\
l
\end{array}\right)(n-l) ! G_{n-k}^{-v, m}(a, b){ }_{L} G_{k-l}^{v, m}(a, b ; x, y, z) H_{l}(-y,-z) .
\end{aligned}
$$

Proof. Differentiating both sides of (16) $k$ times with respect to $y$ and $z$ and then equating the coefficient of $t^{n}$, we obtain (21) and (22) respectively.

Differentiating both sides of (16), with respect to $y$ and $z$, $k$ times and $l$ times, respectively, and equating the coefficient of $t^{n}$, we obtain (23).

Using the right-hand side of (13) and (15) in (17), with the help of (18) for $k=1$, we obtain (24).

Using equations (1), (4), and (13) in (17) with the help of (18) for $k=1$ and then equating the coefficient of $t^{n}$, we obtain (25).

Using (5) and (13) in (17) with the help of (18) for $k=2$, we obtain (26). From (17), we have

$$
\begin{aligned}
e^{y t+z t^{2}} C_{0}(x t)= & {\left[1-2(a+b) t+t^{m}(2 a b+1)\right]^{v} } \\
& \sum_{n=0}^{\infty}{ }_{L} G_{n}^{v, m}(a, b ; x, y, z) t^{n} .
\end{aligned}
$$

Using (13) and (15) in (29), we derive (27).

Again, from (17), we get

$$
\begin{aligned}
C_{0}(x t)= & e^{-y t-z t^{2}}\left[1-2(a+b) t+t^{m}(2 a b+1)\right]^{v} \\
& \sum_{n=0}^{\infty}{ }_{L} G_{n}^{v, m}(a, b ; x, y, z) t^{n} .
\end{aligned}
$$

Using (1), (4), and (13) in (30) and rearranging the equations, we get the desired result in (28).

\section{Differential-Recursive Relations}

In this section of the paper, we have derived few differentialrecursive relations involving the Laguerre-based generalized Humbert polynomial in (16), generalized class of Humbert polynomials in (13), and Hermite-Laguerre polynomial in (15).

Theorem 2. Let $a, b, x, y, z, v \in \mathbb{C}, m \in \mathbb{N}$, and $k \in \mathbb{N}_{0}$. Then, the following results hold: 


$$
\begin{aligned}
n y_{H} L_{n}(x, y, z)+2 n(n-1) z_{H} L_{n-2}(x, y, z)+x \frac{\partial}{\partial x}{ }_{H} L_{n}(x, y, z) & \\
= & n ! \sum_{k=0}^{n} G_{n-k}^{-v, m}(a, b)_{L} G_{n}^{v, m}(a, b ; x, y, z), \quad(n \in \mathbb{N} \backslash\{1\}), \\
= & n ! \sum_{k=0}^{n} G_{n-k}^{-v, m}(a, b)_{L} G_{k}^{v, m}(a, b ; x, y, z), \quad(n \in \mathbb{N} \backslash\{1\}), \\
{ }_{H} L_{n}(x, y, z) & =\sum_{k=0}^{n}(n-2 k) ! G_{n-k}^{-v, m}(a, b) \frac{\partial^{k}}{\partial z^{k}} G_{k}^{v, m}(a, b ; x, y, z), \quad\left(n, k \in \mathbb{N}_{0}, 2 k \leq n\right) . \\
{ }_{H} L_{n}(x, y, z) & =\sum_{k=0}^{N}(n-k) ! G_{n-k}^{-v, m}(a, b) \frac{\partial^{k}}{\partial y^{k}} G_{k}^{v, m}(a, b ; x, y, z), \quad\left(n, k \in \mathbb{N}_{0}, k \leq n\right) .
\end{aligned}
$$

Proof: . Using (13) and (17), we get

Differentiating both sides with respect to $t$, we obtain

$$
e^{y t+z t^{2}} C_{0}(x t)=\sum_{n=0}^{\infty} \sum_{k=0}^{n} G_{n-k}^{-v, m}(a, b)_{L} G_{k}^{v, m}(a, b ; x, y, z) t^{n} .
$$

$$
y e^{y t+z t^{2}} C_{0}(x t)+2 z t e^{y t+z t^{2}} C_{0}(x t)+e^{y t+z t^{2}} \frac{\partial}{\partial t} C_{0}(x t)=\sum_{n=1}^{\infty} \sum_{k=0}^{n} G_{n-k}^{-v, m}(a, b)_{L} G_{k}^{v, m}(a, b ; x, y, z) t^{n-1} .
$$

Multiplying both sides of (35) by $t$ and then using

we get

$$
t \frac{\partial}{\partial t} C_{0}(x t)=x \frac{\partial}{\partial x} C_{0}(x t)
$$

$$
y t e^{y t+z t^{2}} C_{0}(x t)+2 z t^{2} e^{y t+z t^{2}} C_{0}(x t)+e^{y t+z t^{2}} x \frac{\partial}{\partial x} C_{0}(x t)=\sum_{n=0}^{\infty} \sum_{k=0}^{n} G_{n-k}^{-v, m}(a, b)_{L} G_{k}^{v, m}(a, b ; x, y, z) t^{n}
$$

Applying (15), we get

$$
y \sum_{n=0}^{\infty}{ }_{H} L_{n}(x, y, z) \frac{t^{n+1}}{n !}+2 \sum_{n=0}^{\infty}{ }_{H} L_{n}(x, y, z) \frac{t^{n+2}}{n !}+x \frac{\partial}{\partial x}\left\{e^{y t+z t^{2}} C_{0}(x t)\right\}=\sum_{n=0}^{\infty} \sum_{k=0}^{n} G_{n-k}^{-v, m}(a, b)_{L} G_{k}^{v, m}(a, b ; x, y, z) t^{n}
$$

Equating the coefficients of $t^{n}$, we derive (31).

Differentiating both sides of (34) $k$ times with respect to $z$ and $y$ and then comparing the coefficient of $t^{n}$, we obtain the desired results in (32) and (33), respectively.

\section{Symmetry Identities}

In this section, we derive few additional symmetric identities for Laguerre-based generalized Humbert polynomials which are summarized in Theorem 3. 
Theorem 3. For $a, b, x, y, y_{1}, y_{2}, z, z_{1}, z_{2}, v, v_{1}, v_{2} \in \mathbb{C}$, $m \in \mathbb{N}$, and $n \in \mathbb{N}_{0}$, the following identities hold:

$$
\begin{aligned}
& { }_{L} G_{n}^{\nu_{1}+\nu_{2}, m}(a, b ; x, y, z)=\sum_{r=0}^{n} G_{n-r}^{\nu_{1}, m}(a, b){ }_{L} G_{r}^{\nu_{2}, m}(a, b ; x, y, z) \\
& =\sum_{r=0}^{n} G_{n-r}^{v_{2}, m}(a, b)_{L} G_{r}^{\nu_{1}, m}(a, b ; x, y, z) \\
& { }_{L} G_{n}^{\nu, m}\left(a, b ; x, y_{1}+y_{2}, z\right)=\sum_{r=0}^{n} \frac{y_{1}^{n-r}}{(n-r) !}{ }^{L} G_{r}^{\nu, m}\left(a, b ; x, y_{2}, z\right), \\
& =\sum_{r=0}^{n} \frac{y_{2}^{n-r}}{(n-r) !}{ }^{L} G_{r}^{v, m}\left(a, b ; x, y_{1}, z\right) \\
& { }_{L} G_{n}^{\nu, m}\left(a, b ; x, y, z_{1}+z_{2}\right)=\sum_{r=0}^{[n / 2]} \frac{z_{1}^{r}}{(r) !^{L}} G_{n-2 r}^{\nu_{2}, m}\left(a, b ; x, y, z_{2}\right) \\
& =\sum_{r=0}^{[n / 2]} \frac{z_{z}^{r}}{(r) !}{ }_{L} G_{n-2 r}^{\nu_{2}, m}\left(a, b ; x, y, z_{1}\right) \\
& { }_{L} G_{n}^{v_{1}+v_{2}, m}\left(a, b ; x, y_{1}+y_{2}, z\right)=\sum_{r=0}^{n} \sum_{l=0}^{r} \frac{\left(y_{1}\right)^{n-r}}{(n-r) !} G_{n-r}^{v_{1}, m}(a, b){ }_{L} G_{l}^{v_{2}, m}\left(a, b ; x, y_{2}, z\right) \\
& =\sum_{r=0}^{n} \sum_{l=0}^{r} \frac{\left(y_{1}\right)^{n-r}}{(n-r) !} G_{n-r}^{v_{2}, m}(a, b)_{L} G_{l}^{v_{1}, m}\left(a, b ; x, y_{2}, z\right) \\
& =\sum_{r=0}^{n} \sum_{l=0}^{r} \frac{\left(y_{2}\right)^{n-r}}{(n-r) !} G_{n-r}^{\nu_{2}, m}(a, b){ }_{L} G_{l}^{\nu_{1}, m}\left(a, b ; x, y_{1}, z\right) \\
& =\sum_{r=0}^{n} \sum_{l=0}^{r} \frac{\left(y_{2}\right)^{n-r}}{(n-r) !} G_{n-r}^{\nu_{1}, m}(a, b){ }_{L} G_{l}^{\nu_{2}, m}\left(a, b ; x, y_{1}, z\right) \text {, } \\
& { }_{L} G_{n}^{v_{1}+v_{2}, m}\left(a, b ; x, y, z_{1}+z_{2}\right)=\sum_{r=0}^{n} \sum_{l=0}^{[r / 2]} \frac{\left(z_{1}\right)^{l}}{(l) !} G_{n-r}^{v_{1}, m}(a, b){ }_{L} G_{r-2 l}^{\nu_{2}, m}\left(a, b ; x, y, z_{2}\right), \\
& =\sum_{r=0}^{n} \sum_{l=0}^{[r / 2]} \frac{\left(z_{1}\right)^{l}}{(l) !} G_{n-r}^{\nu_{2}, m}(a, b)_{L} G_{r-2 l}^{\nu_{1}, m}\left(a, b ; x, y, z_{2}\right) \\
& =\sum_{r=0}^{n} \sum_{l=0}^{[r / 2]} \frac{\left(z_{2}\right)^{l}}{(l) !} G_{n-r}^{v_{1}, m}(a, b)_{L} G_{r-2 l}^{\nu_{2}, m}\left(a, b ; x, y, z_{1}\right) \\
& =\sum_{r=0}^{n} \sum_{l=0}^{[r / 2]} \frac{\left(z_{2}\right)^{l}}{(l) !} G_{n-r}^{\nu_{2}, m}(a, b)_{L} G_{r-2 l}^{\nu_{1}, m}\left(a, b ; x, y, z_{1}\right) \text {, } \\
& { }_{L} G_{n}^{\nu, m}\left(a, b ; x, y_{1}+y_{2}, z_{1}+z_{2}\right)=\sum_{r=0}^{n} \frac{H_{r}\left(y_{2}, z_{2}\right)}{r !}{ }_{L} G_{n-r}^{\nu, m}\left(a, b ; x, y_{1}, z_{1}\right), \\
& =\sum_{r=0}^{n} \frac{H_{r}\left(y_{1}, z_{2}\right)}{r !}{ }_{L} G_{n-r}^{v, m}\left(a, b ; x, y_{2}, z_{1}\right) \\
& =\sum_{r=0}^{n} \frac{H_{r}\left(y_{1}, z_{1}\right)}{r !}{ }_{L} G_{n-r}^{\nu, m}\left(a, b ; x, y_{2}, z_{2}\right) \\
& =\sum_{r=0}^{n} \frac{H_{r}\left(y_{2}, z_{1}\right)}{r !}{ }_{L} G_{n-r}^{\nu, m}\left(a, b ; x, y_{1}, z_{2}\right)
\end{aligned}
$$




$$
\begin{aligned}
{ }_{L} G_{n}^{\nu_{1}+v_{2}, m}\left(a, b ; x, y_{1}+y_{2}, z_{1}+z_{2}\right) & =\sum_{r=0}^{n} \sum_{l=0}^{r} \frac{H_{r}\left(y_{2}, z_{2}\right)}{(r-l) !} G_{n-r}^{\nu_{1}, m}(a, b)_{L} G_{l}^{\nu_{2}, m}\left(a, b ; x, y_{1}, z_{1}\right), \\
& =\sum_{r=0}^{n} \sum_{l=0}^{r} \frac{H_{r}\left(y_{1}, z_{2}\right)}{(r-l) !} G_{n-r}^{\nu_{1}, m}(a, b)_{L} G_{l}^{\nu_{2}, m}\left(a, b ; x, y_{1}, z_{2}\right) \\
& =\sum_{r=0}^{n} \sum_{l=0}^{r} \frac{H_{r}\left(y_{2}, z_{2}\right)}{(r-l) !} G_{n-r}^{\nu_{2}, m}(a, b)_{L} G_{l}^{\nu_{1}, m}\left(a, b ; x, y_{1}, z_{1}\right) \\
& =\sum_{r=0}^{n} \sum_{l=0}^{r} \frac{H_{r}\left(y_{1}, z_{2}\right)}{(r-l) !} G_{n-r}^{\nu_{2}, m}(a, b)_{L} G_{l}^{\nu_{1}, m}\left(a, b ; x, y_{1}, z_{2}\right) .
\end{aligned}
$$

Proof. Using polynomials involved in (1), (13), and (17) along with equation (18), we can prove the identities mentioned in Theorem 3.

\section{Implicit Summation Formula}

The following theorem establishes the implicit summation formula of Laguerre-based generalized Humbert polynomial.
Theorem 4. Let $a, b, c, x, y, z, v, \alpha \in \mathbb{C}, \quad m \in \mathbb{N}$, and $k, n, i, j \in \mathbb{N}_{0}$. Then,

$$
{ }_{L} G_{k+n}^{\nu, m}(a, b, c ; x, \alpha, z)=\sum_{i=0}^{n} \sum_{j=0}^{k}\left(\begin{array}{c}
n \\
i
\end{array}\right)\left(\begin{array}{c}
k \\
j
\end{array}\right)(\alpha-y)^{i+j} \log ^{i+j}(c){ }_{L} G_{k+n-i-j}^{v, m}(a, b, c ; x, y, z) .
$$

Proof. Replacing $t$ by $t+u$ in (16), we have

$$
\left[1-2(a+b)(t+u)+(t+u)^{m}(2 a b+1)\right]^{-v} c^{z(t+u)^{2}} C_{0}(x(t+u))=c^{-y(t+u)} \sum_{n=0}^{\infty}{ }_{L} G_{n}^{v, m}(a, b, c ; x, y, z)(t+u)^{n}
$$

Using the binomial expansion of $(t+u)^{n}$ with the help of (19), we get

$$
\left[1-2(a+b)(t+u)+(t+u)^{m}(2 a b+1)\right]^{-v} c^{z(t+u)^{2}} C_{0}(x(t+u))=c^{-y(t+u)} \sum_{k, n=0}^{\infty}(k+n) ! G_{L}^{v, m}(a, b, c ; x, y, z) \frac{t^{n}}{n !} \frac{u^{k}}{k !}
$$

Multiplying both the sides of (48) with $c^{\alpha(t+u)}$, we get

$$
c^{(\alpha-y)(t+u)} \sum_{k, n=0}^{\infty}{ }_{L} G_{k+n}^{v, m}(a, b, c ; x, y, z) \frac{t^{n}}{n !} \frac{u^{k}}{k !}=\sum_{k, n=0}^{\infty}{ }_{L} G_{k+n}^{v, m}(a, b, c ; x, y, z) \frac{t^{n}}{n !} \frac{u^{k}}{k !} .
$$

On expanding the exponential function with the aid of (20), we get the following equation:

$$
c^{(\alpha-y)(t+u)}=\sum_{N=0}^{\infty}(\alpha-y)^{N} \log ^{N} c \frac{(t+u)^{N}}{N !}=\sum_{i, j=0}^{\infty} \frac{(\alpha-y)^{i+j} \log ^{i+j} c}{i ! j !} t^{i} u^{j} .
$$


Substituting equation (50) in (49), we get a quadruple series as follows:

$$
\sum_{n, k=0}^{\infty} \sum_{i=0}^{n} \sum_{j=0}^{k} \frac{(\alpha-y)^{i+j} \log ^{i+j} c}{i ! j !}{ }_{L} G_{k+n-i-j}^{v, m}(a, b, c ; x, y, z) \frac{t^{n}}{(n-i) !} \frac{u^{k}}{(k-j) !}=\sum_{k, n=0}^{\infty}{ }_{L} G_{k+n}^{v, m}(a, b, c ; x, \alpha, z) \frac{t^{n}}{n !} \frac{u^{k}}{k !}
$$

Comparing the coefficient of $t^{n} u^{k}$ on both sides in (51), we get the desired result in (46).

\section{Special Cases}

Let us now investigate few special cases of Laguerre-based generalized Humbert polynomial summarized as follows:

Case 1:

$$
{ }_{L} G_{n}^{0, m}(a, b, e ; x, y, z)={ }_{H} L_{n}(x, y, z),
$$

where ${ }_{H} L_{n}(x, y, z)$ is the Hermite-Laguerre polynomial (see [6]).

Case 2:

$$
{ }_{L} G_{n}^{0, m}(a, b, e ; 0, y, z)=H_{n}(y, z),
$$

where $H_{n}(y, z)$ is the Hermite polynomial (see [1]).

Case 3:

$$
{ }_{L} G_{n}^{\nu, m}(a, b, e ; 0,0,0)=G_{n}^{\nu, m}(a, b),
$$

where $G_{n}^{\nu, m}(a, b)$ is the generalized Humbert polynomial (see $[10,13]$ ).

Case 4:

$$
{ }_{L} G_{n}^{\nu, 2}(a, 0, e ; 0,0,0)=C_{n}^{\nu}(x)
$$

where $C_{n}^{v}(a)$ is the Gegenbauer polynomial (see $[13,14])$.

Case 5: letting $v=1 / 2$ and $v=1$ in equation (55), respectively, we get

$$
{ }_{L} G_{n}^{1,2}(a, 0, e ; 0,0,0)=U_{n}(a),
$$

where $U_{n}(a)$ is the Chebyshev polynomial of second kind (see $[13,14])$. Also,

$$
{ }_{L} G_{n}^{1 / 2,2}(a, 0, e ; 0,0,0)=P_{n}(a),
$$

where $P_{n}(a)$ is the Legendre polynomial of first kind (see $[13,14])$.

Case 6:

$$
{ }_{L} G_{n}^{v, 2}(a, 0, e ; 0,2 a,-1)={ }_{H} C_{n}^{v}(a),
$$

where ${ }_{H} C_{n}^{v}(a)$ is the Hermite-Gegenbauer polynomial (see [14]).

Case 7: letting $v=1 / 2$ and $v=1$ in equation (58), respectively, we get

$$
{ }_{L} G_{n}^{1 / 2,2}(a, 0, e ; 0,2 a,-1)={ }_{H} P_{n}(a),
$$

where ${ }_{H} P_{n}(a)$ is the Hermite-Legendre polynomial of one variable (see [14]). Also,

$$
{ }_{L} G_{n}^{1,2}(a, 0, e ; 0,2 a,-1)={ }_{H} U_{n}(a),
$$

where ${ }_{H} U_{n}(x)$ is the Hermite-Chebyshev polynomial of one variable (see [14]).

Case 8:

$$
{ }_{L} G_{n}^{\nu, m}(a, b, e ; 0, y, z)={ }_{H} G_{n}^{\nu, m}(a, b ; y, z),
$$

where ${ }_{H} G_{n}^{\nu, m}(a, b, y, z)$ is the Hermite-based generalized Humbert polynomial.

Case 9:

$$
{ }_{L} G_{n}^{1 / 2, m}(a, b, e ; x, y, z)={ }_{H} P_{n}^{\nu, m}(a, b ; x, y, z),
$$

where ${ }_{H} P_{n}^{v, m}(a, b ; x, y, z)$ is the Laguerre-based Legendre polynomials of two variables. Also,

$$
{ }_{L} G_{n}^{1, m}(a, b, e ; x, y, z)={ }_{H} U_{n}^{\nu, m}(a, b ; x, y, z),
$$

where ${ }_{H} U_{n}^{\nu, m}(a, b ; x, y, z)$ is the Laguerre-based Chebyshev polynomials of two variables.

Using these special cases to the identities derived in previous sections, we can derive the corresponding identities. To state an example, we have derived an implicit summation formula for Hermite-based generalized Humbert polynomial in the following corollary.

Corollary 1. Let $a, b, y, z, v, \alpha \in \mathbb{C}, \quad m \in \mathbb{N}$, and $k, n, i, j \in \mathbb{N}_{0}$. Then,

$$
{ }_{H} G_{k+n}^{v, m}(a, b ; \alpha, z)=\sum_{i=0}^{n} \sum_{j=0}^{k}\left(\begin{array}{l}
n \\
i
\end{array}\right)\left(\begin{array}{c}
k \\
j
\end{array}\right)(\alpha-y)^{i+j}{ }_{H} G_{k+n-i-j}^{v, m}(a, b ; y, z) .
$$

\section{Conclusion}

This study has defined a new polynomial class, namely, Laguerre-based generalized Humbert polynomials. We have derived recursive relations, additional symmetry identities, and implicit summation formulas for these special functions. We have also defined few essential special cases of this class; a few of them are Laguerre-based Legendre, Laguerre-based Chebyshev, Hermite-based generalized Humbert 
polynomial etc. Using these special cases to the identities derived, we get the corresponding identities.

\section{Data Availability}

No data were used to support this study.

\section{Conflicts of Interest}

The authors declare that they have no conflicts of interest.

\section{References}

[1] E. T. Bell, "Exponential polynomials," The Annals of Mathematics, vol. 35, no. 2, pp. 258-277, 1934.

[2] E. D. Rainville, Special Functions, Macmillan Company, New York, NY, USA, 1960.

[3] M. A. Bassuony, W. M. Abd-Elhameed, E. H. Doha, and Y. H. Youssri, "A legendre-laguerre-galerkin method for uniform euler-Bernoulli beam equation," East Asian Journal on Applied Mathematics, vol. 8, no. 2, pp. 280-295, 2020.

[4] E. H. Doha and Y. H. Youssri, "On the connection coefficients and recurrence relations arising from expansions in series of modified generalized Laguerre polynomials: applications on a semi-infinite domain," Nonlinear Engineering, vol. 8, no. 1, pp. 318-327, 2019.

[5] G. Dattoli and A. Torre, "Operatorial methods and two variable Laguerre polynomials," Atti Acad. Torino, vol. 132, pp. 1-7, 1998.

[6] N. U. Khan, T. Usman, and J. Choi, "Certain generating function of Hermite-Bernoulli-Laguerre polynomials," Far East Journal of Mathematical Sciences (FJMS), vol. 101, no. 4, pp. 893-908, 2017.

[7] N. Khan, T. Usman, and J. Choi, "A new class of generalized Laguerre-Euler polynomials," Revista de la Real Academia de Ciencias Exactas, Físicas y Naturales. Serie A. Matemáticas, vol. 113, no. 2, pp. 861-873, 2019.

[8] N. U. Khan, T. Usman, and W. A. Khan, "A new class of Laguerre based generalized Hermite-Euler polynomials and its properties," Kragujevac Journal of Mathematics, in press, 2018.

[9] G. V. Milovanovi'c and G. B. Djordjevi'c, "On some properties of Humberts polynomials-I," Fibonacci Quart, vol. 25, pp. 356-360, 1987.

[10] G. B. Djordjevi'c, "A generalization of Gegenbauer polynomial with two variables," Indian Journal of Pure and Applied Mathematics, To appear in.

[11] J.-S. Choi, "Notes on formal manipulations of double series," Communications of the Korean Mathematical Society, vol. 18, no. 4, pp. 781-789, 2003.

[12] H. M. Srivastava and H. L. Manocha, A Treatise on Generating Functions, Ellis Horwood Limited, Chichester, UK, 1984.

[13] M. A. Pathan and M. A. Khan, "On polynomials associated with Humberts polynomials," Publications de l'Institut Mathématique, (Beograd), vol. 62, no. 76, pp. 53-62, 1997.

[14] M. A. Pathan and W. Khan, "On a class of humbert-hermite polynomials," Novi Sad Journal of Mathematics, 2019, Preprints. 